\title{
Identifying Exosomes as a Messenger Unit During Heterochronic Parabiosis for Amelioration of Huntington's Disease
}

Mijung Lee

Seoul National University Hospital

Wooseok Im

Rosetta Exosome Inc.

Manho Kim ( $\sim$ kimmanho@snu.ac.kr)

Seoul National University Hospital https://orcid.org/0000-0002-0277-6326

Research article

Keywords: Neuroscience, Protein Metabolism, heterochronic parabiosis

Posted Date: July 1st, 2020

DOI: https://doi.org/10.21203/rs.3.rs-36871/v1

License: (c) (i) This work is licensed under a Creative Commons Attribution 4.0 International License.

Read Full License 


\section{Abstract}

Background:Huntington's disease (HD) starts its pathology long before clinical manifestation, however, there is no therapy to cure it completely and only a few studies have been reported for delaying the progression of HD. We demonstrated the blood sharing effect by heterochronic parabiosis in HD and explored the underlying mechanism for transferring positive factors in the young blood serum.A shared blood circulation by heterochronic parabiosis has improved behavioral performance and HD pathology through mediation of mitochondria dysfunction and cell death. Furthermore, the messenger unit for the effective components in young blood is identified for the first time to the best of our knowledge.

Methods:R6/2 mice were surgically connected with young wild-type mice $(n=13)$, old wild-type mice $(n=8)$, or R6/2 mice $(n=6)$ to examine the effect of heterochronic parabiosis.Parabionts composed of 5 - to 6week-old transgenic and wild-type mice were observed for 6 weeks in a single cage. The in vitro cellular model of HD cells were treated by the $200 \mu \mathrm{g} / \mathrm{mlblood}$ serum of the young or old mice, and by the exosomes isolated from thereof. Thein vitro cellular model of HD were developed by differentiating neural stem cells cultured from SVZ of the brain.

Results:After the heterochronic parabiosis, the weight loss and survival of HD mice was improved, and also, mutant Huntingtin aggregation (EM48 $p<0.005$ ), improvement of mitochondria dysfunction (PGC-1a $p<0.05, p-C R E B / C R E B ~ p<0.005)$, cell death (p53 p<0.05, Bax p<0.05, Cleaved-caspase3 $p<0.05$ ), and cognition ( $D C X p<0.5)$ showed a near complete restoration. In addition, treatment of exosomes from young blood serum to thein vitro cellular model of HD improved mutant Huntingtin aggregation (EM48 $p<0.05$ ), mitochondria biogenesis ( $p$-CREB/CREB $p<0.005$ ), cell death ( $p 53 p<0.05$, Bax $p<0.005$, Cleavedcaspase3 $p<0.05, B c l-2 p<0.05)$, and cell proliferation (WST-1 $p<0.005)$.

Conclusions:We found that the overall pathology of HD is improved by the shared blood circulation through heterochronic parabiosis, furthermore, we demonstrated that the exosomes are messengers for transferring positive factors, showing the potential of exosomes from young bloodfor the amelioration of HD.

\section{Background}

Huntington's disease(HD) is a fatal, progressive neurodegenerative disease with an autosomal dominant inheritance, characterized by chorea, involuntary movements of the limbs and cognitive impairment.[1-3] HD is caused by an expanded CAG repeat in the HD gene on chromosome 4.[1,3,4] This mutation produced an extended $\mathrm{N}$-terminal polyglutamin stretch in the huntingtin $(\mathrm{Htt})$ protein leading to intracellular accumulation and aggregation of mutant huntingtin ( $\mathrm{mHtt}$ ).[1] Accumulation of $\mathrm{mHtt}$ aggregates causes striatal cell death through transcriptional dysregulation, activation of intrinsic apoptosis pathways, mitochondrial dysfunction, and alters protein-protein interactions. $[5,6]$ To present date, there isno promising cure or beneficial treatment for the disease and developing therapies are in high priority.[7, 8] 
Several studies in other neurodegenerative diseases like AD have shown that exposure to a young blood circulation through heterochronic parabiosis, which is a surgical union of 2 organisms of different phenotype that leading to the formation of a vascular anastomosis and a shared circulatory system between two mice,[9] reverses cognitive deficits that is observed with normal aging. Although the idea of adopting heterochronic parabiosisto the HD seems pretty straightforward, however, it has not been reported so far inR6/2 mice. It is becausethe mouse model for $\mathrm{HD}$, are highly vulnerable to stress and at 3 to 4 months of age, they develop trimming and cause death by muscle loss.[10,11]

Also, a thorough understanding for the transportation mechanism of positive factors from the young blood to the old duringheterochronic parabiosis is necessary in order to adoptthe heterochronic parabiosis as the cure for HD. However, previous studies have only focused on which factors of 'young blood' have a positive effect on neurodegenerative disease.[9, 12-14]There wasonly vague understandings like the diffusion of blood that how the positive factors are transported, because the transportation process is nanoscale, which makes it difficult to identify the messenger unit.

We have focused on exosomes which are the smallest membranous vesicles $(40-100 \mathrm{~nm})$ that has cargo ability for intercellular matter exchange.[15-17]Exosomes are generated via the inward budding of endosomes, to form multivesicular bodies (MVBs) that fuse with the membranes to release exosomes into the surrounding environment.[18, 19]Exosomes, depending on their parental origin, contain a variety of proteins, lipids, non-coding RNAs, mRNA, and miRNA, collectively termed as "cargo" contains. Due to their cargo ability, exosomes represent a novel form of intracellular communication among cells without cell-to-cell direct contact. Exosomes are selectively taken up by the surrounding or distal cells and can reprogram the recipient cells due to their active cargo content.[20,21]

By minimizing the damage of parabiosis surgery, we were able to make R6/2 mice survive over 12 month in the case of control group, and over 17 month in the case of heterochronic parabiosis group, successively producing the HD R6/2 animal model for the heterochronic parabiosis for the first time.By the heterochronic parabiosis of R6/2 mice, we foundthat young blood has positive factors in improving the HD pathology, and the identical results could be obtained by processingthe exosomes extracted from the young seruminto in vitro model. We discovered that the exosomes serve as a messenger unit for the

positive factor of young serum during the heterochronic parabiosis. It could lead to a development of potential small molecule in exosome interventions, and a group of soluble factors in exosome targeting several pathways may help therapeutic benefits for HD.

\section{Methods}

\section{Experimental model of HD}

All experimental animal procedures performed were approved by the Institutional Animal Care and Use Committee (IACUC, Approval number: 16-0043-C2A1) of Seoul National University Hospital, which was accredited by the Association for the Assessment and Accreditation of Laboratory Animal Care International. Transgenic R6/2 (B6CBA-Tg(HDexon1)62Gpb/1J, 111 CAGs) and their WT littermates used 
in this study were purchased from Jackson Laboratory (Bar Harbor, ME, USA). Mice used in this study were zQ175 KI heterozygous (CHDI-81003003) or WT littermates on a C57BL/6J background strain obtained from the CHDI colony at Jackson Laboratories (Bar Harbor, ME) or bred in-house at PsychoGenics, Inc. (Tarrytown, NY). ZQ175 mice, originating from the CAG 140 mice (from germline CAG expansion) were heterozygous and wild-type mice were generated by crossing heterozygous ZQ175 mice on a C57BL/6J background.The R6/2 transgenic mice model expresses exon 1 of a human $\mathrm{mHtt}$ and is the most widely used animal model for studying HD. These mice were obtained by crossing ovarian transplant hemizygote females with B6CBAF1/J males. R6/2 Miceand ZQ175 were bred the Seoul National University Hospital under specific pathogen-free conditions and mice homozygous offspring of heterozygous matings were identified by polymerase chain reaction (PCR) typing of tail-tip genomic DNA. The mice were house in groups with ad libitum access to food and water and a 12 hours light / 12 hours dark cycle. Mouse body weight was recorded weekly.

\section{Procedures for parabiosis}

Parabiosis were subjected to parabiotic surgery using methods adapted from JOVE, Bunster and Meyer. Mice were anesthetized with Zoletil/Rompun intraperitoneal (i.p.)and all surgical procedures were performed under general anesthesia. Operative sides were shaved and sterilized. Lateral skin was opened from hip to shoulder and freed of attached tissue. Opposing muscle and perineum was sutured with 4-0 chromic gut (Roboz, Rockville, MD), and corresponding skin was joined with 9-mm wound clips (Fisher Scientific, Houston, TX). Transgenic R6/2 (B6CBA-Tg(HDexon1)62Gpb/1J, 111 CAGs) disease phenotype appears at 8 weeks of age. Pairs of 5- to 6-week-old Transgenic HD and WT, sex-matched R6/2 mice were housed together in a single cage for 6 weeks. Pairs of 8- to 10-week-old and 28- to 30-week-old, sexmatched ZQ175 (C57BL/6J B6.129S1- Htt<tm1 Mfc>/190JChdi) mice were housed together in a single cage for 16 weeks.

\section{BrdU administration and immunohistochemistry}

To demonstrate a connected circulation between prabionts, BrdU was injected into one mice. The formation of shared blood circulation between the parabiontic animals was tested by injection of BrdU $(150 \mathrm{mg} / \mathrm{kg}$, Sigma-Aldrich) to the intraperitoneal administration of one of the parabionts after 2 weeks post-surgically and the pair was killed after 4 weeks. Mice were anesthetized and perfused through the heart with $10 \mathrm{ml}$ of cold saline and $4 \%$ paraformaldehyde in $0.1 \mathrm{M}$ PBSat 12 weeks of age. Brains were removed from the skull, cryoprotected in $30 \%$ sucrose at $4^{\circ} \mathrm{C}$, and sectioned $20 \mu \mathrm{m}$. Free-floating sections were washed and followed by incubation in $1.5 \mathrm{M}$ hydrogen chloride at $37^{\circ} \mathrm{C}$ for $30 \mathrm{~min}$. After, the sections were washed in PBS with three times and blocked with normal goat serum, then stained with the BrdU antibody (1:300, Abcam, Cambridge, MA, USA). On the following day, the sections were washed in PBS with three times and incubated with Cy3 conjugated anti-rat IgG(1:100; Jackson immune Research Laboratories) for 2 hours. BrdU (red) or DAPI (blue)-stained cells were identified using an inverted microscope (BX61, Olympus Corporation, Tokyo, Japan).

\section{Weight measurement}


Mice from the R6/2×TG2 KO line and from the zQ175×TG2 KO line were weighed every week for 6 weeks and for 16 weeks, respectively.

\section{Preparation of in vitrocellular model for HD}

A colony was maintained by breeding ovarian transplant R6/2 females (B6CBATg(HDexon1)62Gpb/1J) obtained from the Jack-son Laboratories with B6CBAF1/J males, based on a C57BL/ 6 background. F1 offspring of the first mating generated mice with either the wild-type or R6/2 genotype. Mice were housed under standard conditions ( $12 \mathrm{~h}$ light cycle from 08:00 $\mathrm{h}$ to 20:00 $\mathrm{h}$ ) with adlibitum access to food and water. All animal experiments were studied with the approval of the Institutional Animal Care and Use Committee (IACUC, Approval number: 13-0058-C2A1) of Seoul National University Hospital. We developed an in vitro $\mathrm{HD}$ model by culturing neural stem cells from R6/2 mice. In brief, after sacrificed by $\mathrm{CO}_{2}$ gas, brain tissues of 9-week-old mice were used for primary culture and neural stem cells were isolated by dissection and trypsin treatment. Cells were incubated in culture medium consisting of DMEM/F12 (Invitrogen, Carlsbad, CA, USA), 1\% P/S (penicillin-streptomycin), 2\% B27 Supplement (Gibco BRL, Carlsbad, CA, USA), $10 \mathrm{ng} / \mathrm{mL}$ EGF (Invitrogen, Carlsbad, CA, USA) and $10 \mathrm{ng} / \mathrm{mL}$ bFGF (Invitrogen, Carlsbad, $\mathrm{CA}, \mathrm{USA}$ ) at $37^{\circ} \mathrm{C}$ in a $95 \% \mathrm{O}_{2}, 5 \% \mathrm{CO}_{2}$ humidified atmosphere. neural stem cells were differentiated in the differentiation medium, which was composed of DMEM/F12, $1 \%$ PSA, $2 \%$ B27, and $5 \%$ FBS.

\section{Isolation serum exosome and treatment of young and old serum-exo}

To deplete exosomes from the mice serum, all centrifugation steps were performed at $4{ }^{\circ} \mathrm{C}$. Exosomes were isolated by Exo-quick exosome precipitation solution (System Biosciences, Mountain View, CA), according to manufacturer's specifications. Briefly, the serum $250 \mu \mathrm{l}$ was mixed thoroughly with $63 \mu \mathrm{l}$ of Exo-Quick exosome precipitation solution and centrifuged at $1500 \mathrm{~g}$ for $30 \mathrm{~min}$. The supernatant was then removedand centrifuged at $1500 \mathrm{~g}$ for $5 \mathrm{~min}$ after adding buffer. The remaining exosome pellets were

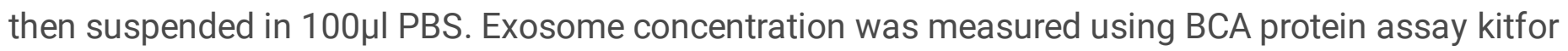
treatment. HD cells were treated with $200 \mu \mathrm{g} / \mathrm{ml}$ of young or old serum-exo and young or old serum at 2 days of differentiation and incubated for 3 days. Control groups were treated with same volume of PBS.

\section{Analysis of $\mathrm{mHtt}$ aggregation in cells}

$\mathrm{mHtt}$ aggregation was quantified by fluorescent immunocytochemistry. Cells were stained with Em48 antibody (1:400, Millipore, Billerica, MA, USA) after fixing with 4\% paraformaldehyde. The cells were then counterstained with DAPI (1:300, Sigma, Deisenhofen, Germany). For the fluorescence staining analysis, we performed three independent experiments and over 500 cells are counted in each group.[22]Em48 (red) or DAPI (blue)-stained cells were counted using an inverted microscope (BX61, Olympus Corporation, Tokyo, Japan).

\section{Tissue preparation and Fluorescent Immunohistochemistry}


For immunohistochemistry,mice were anesthetized and perfused through the heart with $10 \mathrm{ml}$ of cold saline and $4 \%$ paraformaldehyde in $0.1 \mathrm{M}$ PBS at 12 weeks of age. Brains were removed from the skull, cryoprotected in $30 \%$ sucrose at $4^{\circ} \mathrm{C}$, and sectioned $20 \mu \mathrm{m}$. Free-floating sections were washed and blocked with normal goat serum, then stained with the Em48 antibody (1:300, Millipore, Billerica, MA, USA).On the following day, the sections were washed in PBS with three times and incubated with Cy3 conjugated anti-mouse IgG (1:100; Jackson immune Research Laboratories) for 2hours. EM48 (red) or DAPI (blue)-stained cells were identified using an upright microscope (Ni-E, Nikon Corporation, Tokyo, Japan).[23, 24]

\section{DCX and BRDU Immunohistochemistry}

Free-floating sections were washed and followed by incubation in $1.5 \mathrm{M}$ hydrogen chloride at $37^{\circ} \mathrm{C}$ for 30 min. After, the sections were washed in PBS with three times and blocked with normal goat serum, then stained with the DCX antibody (1:200; Santa Cruz, CA, USA) and BrdU antibody (1:300, Abcam, Cambridge, MA, USA). On the following day, the sections were washed in PBS with three times and incubated with FITC conjugated anti-rabbit IgG and Cy3 conjugated anti-rat IgG (1:100; Jackson immune Research Laboratories) for 2hours. DCX (fitc) and BrdU (red) or DAPI (blue)-stained cells were identified using an inverted microscope (BX61, Olympus Corporation, Tokyo, Japan).

\section{Protein extraction and western blot analysis}

Brains of R6/2 mice and ZQ175 were isolated, immediately frozen on liquid nitrogen, and stored at $-80^{\circ} \mathrm{C}$ until protein extraction. Cultured HD cells were washed and harvested in PBS (phosphate buffered saline, WelGene, Daegu, Korea)using a cell scraper. Protein extracts were prepared using RIPA buffer (Radio immunoprecipitation assay buffer, Thermo-Scientific, Waltham, MA, USA)containing freshly added protease inhibitor and phosphatase inhibitor (Roche, NJ, USA). The protein content was determined using a BCA (Bicinchoninic acid assay) protein assay kit (Pierce, Rockford, IL, USA). Forty micrograms of protein samples were separated by sodium dodecyl sulfate-polyacrylamide gel electrophoresis (SDSPAGE, 4-15\% Novex NuPage Bis-Tris gel, Invitrogen, Mount Waverley, Australia) and $10 \%$ or $15 \%$ SDSPAGE and transferred onto polyvinylidene fluoride membrane (PVDF, Millipore, Bedford, MA, USA). Blocking with $5 \%$ non-fat dried milk dissolved in $1 \times$ TBST (Tris-buffered saline with $0.1 \% \mathrm{v} / \mathrm{v}$ Tween-20) for $1 \mathrm{~h}$ at room temperature. Blots were then incubated at $4{ }^{\circ} \mathrm{C}$ overnight with primary antibodies diluted as recommended in the manufacturer's instructions. The following primary antibodies were used: antiPGC1a (1:200; Santa Cruz), p-CREB (1:1000; Cell-signaling), CREB (1:1000; Cell-signaling), anti-p53 (1:1000; Cell-signaling, MA, USA), anti-Bax (1:200; Santa Cruz), BCL-2 (1:200; Santa Cruz), Caspase3 (1:1000; Cell-signaling), Em48 (1:500, Millipore), DCX antibody (1:500, abcam), and anti- $\beta$-actin (1:200, Santa Cruz). Blots were then incubated with horseradish peroxidase-conjugated secondary anti-mouse or anti-rabbit antibodies (1:3000, GE Healthcare, NJ, USA), and developed using ECL solution (Enhanced chemiluminescenece solution, Advansta, CA, USA). Band intensities were measured using ImageJ software from three independent results normalized by $\beta$-actin (Park, 2015). All western blot figures show the representative one from three separate experiments. 


\section{Cell survival assay}

Cell survival rate was measured by a colorimetric assay using the WST-1 (Roche, Mannheim, Germany) according to manufacturer's instruction. Briefly, cells were seeded in 96-well plates and incubated with young and old serum-exo $200 \mu \mathrm{g} / \mathrm{ml}$ for 72 hours. After 72 hours, WST-1 reagent was added to each well, and cells were incubated at $37^{\circ} \mathrm{C}$ and $5 \% \mathrm{CO} 2$ for $2 \mathrm{~h}$. Absorbance was measured using a plate reader at $450 \mathrm{~nm}$ (reference $650 \mathrm{~nm}$ ) and the result shown represent the averages of four independent experiments.

\section{Flow cytometry}

To analyze the apoptosis population of neuronal stem cells, flow cytometry using annexin V-FITC and propidium iodide (PI) staining was used. Neuronal stem cells were washed and harvested in PBS (phosphate buffered saline, WelGene, Daegu, Korea) using a cell scraper. Cells were counted and $1 \times 10^{6}$ cells were suspended in $1 \mathrm{ml}$ cold binding buffer (10 mM HEPES/ NaOH, pH 7.4, $140 \mathrm{mM} \mathrm{NaCl}$, and 2.5 $\mathrm{mM} \mathrm{CaCl}_{2}$ ). Cells were aliquoted into $1.5 \mathrm{ml}$ tube at $1 \times 10^{5}$ cells per tube, and were incubated with $10 \mu \mathrm{l}$ of annexin V-FITC at room temperature for $30 \mathrm{~min}$ and $2 \mu \mathrm{g} / \mathrm{ml}$ of PI at room temperature for $10 \mathrm{~min}$. After incubation, $400 \mu \mathrm{l}$ of binding buffer was added and flow cytometric analysis was performed (FACS Calibur, BD Bioscience, CA, USA). FITC and PI fluorescences were passed through 520 and $630 \mathrm{~nm}$ bandpass filters, respectively, and the data were analyzed using Flowing Software (www.flowingsoftware.com).

\section{Statistical analysis}

All values indicated in the figures are presented as mean \pm standard error. Results of western blot were analyzed using Student's t-test. A 2-tailed probability value below 0.05 was considered statistically significant. Data were analyzed by SPSS version 17.0 (SPSS Inc., USA).

\section{Results}

\section{Construction of Parabiosis Animal Model for HD and Modulation of HD Pathology}

The R6/2 mice were surgically joined either with another R6/2 mice or with their corresponding wild-type littermate.In addition, WT-WT serve as controls to ensure that the surgical procedures did not cause ectopic mineralization (Fig. 1a).To successively perform the parabiosis of R6/2 mice, minimizing the damage of parabiosis and the stress were essential due to the high vulnerability of R6/2 mice. The binding site and method had a critical influence on the survival of R6/2 parabionts, and we were able to obtain stable R6/2 parabionts for the first time.Suturing the abdomen and the back of the mice was performed through clamping and tying their forelimbs with threads. More details about the methods of parabiosis are demonstrated in the Supplement(Additional file 1: Figure S1, Additional file 2: Figure S2, Additional file 3: Video 1, and Additional file 4: Video 2). The mice were observed periodically as described 
in materials and methods. No obvious signs of stress were noted during observations, after 6 weeks after surgery.

To demonstrate a connected circulation between parabionts, BrdU was injected into Het-WT. Brain from the injected mice and the attached mice in all groups of parabionts showed similar BrdU signals, indicating a joined circulatory system (Fig. 1b). The shared circulation was also demonstrated by examining the genomic DNA of blood cells in paired mice at four weeks after parabiotic surgery. By comparing the genotype of DNA from the Het-WT and the Iso-HD, the two bands that can be seen in IsoHD werealso observed in Het-WT (Fig. 1C).

To evaluate the functional consequences of heterochronic parabiosis, we measured weight loss and lifespan. Phenotypes were examined from the parabionts paired for sixweeks. We also recorded survival,weight loss and lifespan of each group among the same composition of females and males.In contrast to theWT(Old)-HD, WT(Young)-HD showed increased survival (Fig. 1d). Heterochronic parabiosis with the young mice delayed the onset of mortality from 84 to 100 days, the average survival from 102 to 123 days, and the maximum survival from 118 to 145 days $(P<0.05)$. It also reduced tremor (data not shown), improved movement measured(Additional file 5 - 7: Video3 - 5), and improved clasping test(Additional file 8 - 10: Video6 - 8) at 9 and 12 weeks of age.Also, WT(Young)-HD showed delayed progression of weight loss at 12 weeks old $(p<0.01)$ while HD-HD showed gradual weight loss from 10 to 12 weeks of age (Fig. 1e), whileR6/2 mice (with CAG repeats of between 154 and 200) die typically at around 3-4 months of age.The improvement on the behavior (Additional file 11 - 13: Video9 - 11) and the weight loss (Additional file 14: Figure S3)were also shown in ZQ175 mice.

The $\mathrm{R} 6 / 2$ mice showed $\mathrm{mHtt}$ aggregation in striatum and cortex during disease progression. To examine histological changes of the brain, $\mathrm{mHtt}$ aggregation were evaluated at 12 weeks of age. To evaluate effect on $\mathrm{mHtt}$ aggregation, brain was sectioned and sliced tissues were stained with an EM48 antibody which detects aggregation of $\mathrm{mHtt}$, and Het-HD showed reduced $\mathrm{mHtt}$ aggregation in the striatum and cortex (Fig. 1f). Also, we extracted proteins from R6/2 mice brain and $\mathrm{mHtt}$ aggegation were measured by western blotanalysis (Fig. 1g), and WT(Young)-HD showed reduced $\mathrm{mHtt}$ aggregationin the brain.

\section{Modulation of Pathological Phenotypes of HD by Parabiosis}

Dysfunction of CREB-PGC-1 a pathway has been regarded as the key molecule for HD progression. To examine effects of Heterochronic parabiosis on this pathway, 12 weeks old R6/2 mice were paired and western blot analysis was performed aftersix weeks from the surgery. Het-HD showed increased expression of $p$-CREB and PGC-1a ( $p<0.05$ vs. R6/2 control) compared to Iso-HD (Fig. 2a). To investigate whether heterochronic parabiosisprotects against apoptosis, we examined the levels of apoptosis-related proteins by western blotting. p53, Bax, and cleaved caspase-3 levels were lower in the Het-HD than Iso-HD (Fig. 2b). 
Also, $\mathrm{IHC}$ and western blot were performed to examine the improvement of cognitive function. It was found that DCX and BrdU, which are the representative signals for neurogenesis, were increased in Het-HD (Fig. 2c), and the same result was confirmed by western blot (Fig. 2d). It proves that young wild-type blood is also effective in improving cognition. Also, the same result was obtained for the modulation of pathological phenotypes of HD by parabiosis in the case of ZQ175 mice (Additional file $15-16$ : FigureS4 -S5).

\section{Amelioration of $\mathrm{mH} t \mathrm{tt}$ Aggregation by Exosome Treatment}

To construct the in vitro cellular model for HD, neural stem cells were separated from the SVZ which was isolated from the R6/2 mice brain. Neural stem cells showed a spherical shape after the separation, and the expression of $\mathrm{mHtt}$ aggregation protein was not observed. The $\mathrm{mHtt}$ aggregation protein can be seen from 5 to 7 days after the differentiation of the cell, which is expressed as red in the cell nucleus (Additional file 17: FigureS6).To harvest the exosomes from the blood serum, thereare two representative methods for exosome isolation: serial ultracentrifugation and Exo-Quick reagent. Western bolt analyses were performed to determine the expression of exosome-specific markerswhich are CD9, CD63 (tetraspanin proteins) and HSP70. The isolated products expressed all the markers, thus confirming the presence of exosomes (Additional file 18: FigureS7).

As shown earlier in Fig. 1d, paring the HD mice with theyoung wild-type increased survival, whereasparing with old wild-type did not. To investigate the underlying mechanismof age-dependent effect of the parabiosis with WT, the exosomal protein and RNA concentration in young blood and old blood were analyzed. Proteins and total RNA were extracted by protein extraction buffer and total RNA isolation kit, respectively, after isolating exosomes. Higher amount of exosomes, exosomal protein, and RNA levels were observed in young blood (Fig. 3a).

To investigate whether young serum-exosomes has a protective role in $\mathrm{HD}$, we treated an in vitrocellular model for HD, which showed $\mathrm{mHtt}$ aggregations in nucleus after day 7 of induction, with young serumexosomes. Young serum-exosomes $(200 \mu \mathrm{g} / \mathrm{mL})$ was applied to the cells for 3 days after inducing $\mathrm{mHtt}$ aggregation. At day 7 , the control and young serum-exosomes groups were fixed with $4 \%$

paraformaldehyde and stained with the Em48 antibody to detect $\mathrm{mHtt}$ aggregates, with DAPI as a counter stain (Fig. 3b). We counted DAPI(+) and Em48(+) cells in the HDand HD+Young-exo. The ratios of doublepositive cells to $\mathrm{DAPI}(+)$ cells were $20.8 \pm 2.3 \%$ for $\mathrm{HD}$ and $12.1 \pm 0.3 \%$ for $\mathrm{HD}+$ Young-exo. To confirm the reduction of $\mathrm{mHtt}$ aggregates, aggregates were also quantified by western blot (Fig. 3c). In HD+Youngexo, levels of $\mathrm{mHtt}$ aggregates in cells were significantly decreased.

\section{Modulation of Molecular Pathology of HD by Exosome Treatment}

To examine the effects of young serum-exosomeson the p-CREB-PGC1a pathway, cells were treated with control medium or young serum-exosomes for three days after days 2 of differentiation. Treatment with 
young serum-exosomes promoted expression of p-CREB and PGC1a (Fig. 4a). To examine theprotection against apoptosis, the levels of apoptosis-related proteins were evaluated by western blotting. p53, Bax, and cleaved caspase-3 levels were lower in the HD+Young-exo than in the HD (Fig. 4b). Also, to confirm the anti-apoptotic effect of young serum-exosomes, neural stem cellswere differentiated and treated by the young serum-exosomes for 3 days, and flow cytometry analysis was performed using annexin- $V$ and propidium iodide (Fig. 4c). Cell population wasanalyzed as viable/early-apoptotic/lateapoptotic/necrosis, and this calculation was conducted using the annexin- $\mathrm{V}$ and propidium iodide positive cell count. More necrotic population and less viable population were shown in HD,however, $\mathrm{HD}+$ Young-exo showed significant reduction of the apoptotic/necrotic cell population and an increase in the viable cell population.Taken together, young serum-exo treatment resulted in more cell survival and less cell death, accompanied by a reduction of $\mathrm{mHtt}$ aggregation protein, and apoptotic signaling. To examinethe cell survival effects, we investigated the WST-1. The result showed that treatment of young serum-exosomes significantly increases cell survival (Fig. 4d). Young serum-exosomes improves mitochondrial activation and cell survival while old serum-exosomes does not (Additional file 19: Figure S8).

\section{Discussion}

In this study, we demonstrated thatheterochronic parabiosis of HD mice with the young wild-type modulates the body weight loss, $\mathrm{mHtt}$ aggregation, mitochondrial dysfunction, cell death, and cognitive impairment.[1, 10] These are the representative pathologies of HD, thus extending the survival of HD mice. The positive effect ofheterochronic parabiosis originates from the shared blood circulation, $[25,26]$ just like the other neurodegenerative diseases ameliorated by heterochronic parabiosis. $[9,27,28] \mathrm{t}$ is worth noting that the R6/2 mice die typically at around 3-4 months of ageaccompanied by trimming and muscle loss $[10,11]$ and highly vulnerable to stress. Therefore minimizing the damage of parabiosis surgery were indispensableto make stable parabiotic pairs. We were able to obtain stable R6/2 parabionts for the first timeby optimizing the binding site and method, which had a critical influence on the survival of $\mathrm{R} 6 / 2$ parabionts.

As we have already demonstrated thatthe positive factorsexistin the young blood affecting the transgenic ones,[14]it should also exist in the messenger unit in the shared circulatory system. We identified that the exosomes are messenger units for transferring positive factors inside the blood by the in vitro cellular model for HD.Young blood serum as well as serum exosomesalso modulated $\mathrm{mHtt}$ aggregation, mitochondrial dysfunction, cell death, and cell viability.

Although there are several limitations that further study is required to identify the positive factor itselfthat are responsible for elucidating the mechanisms of exosome treatment.[16, 29] However, we demonstrated that exosome showed more than equivalent effect compared to serum, which could lead to the development of the intravenous administration of serum exosome in humans, which is a low risk procedure already offered as a therapy with limited complications,[30,31] and a group of soluble factors in exosome targeting several pathways may help therapeutic benefits. Therefore, itis feasible to test the 
efficacy of young serum exosome in patients with HD and possibly other forms of mitochondria dysfunction and neurodegeneration.

\section{Conclusion}

In summary, our results show that the overall pathology of HD is improved by the shared blood circulation through parabiosis, furthermore, we demonstrated that the exosomes are messenger units for transferring positive factors inside the blood by the in vitro cellular model for HD. Thus, the therapeutic potential of the exosomes from young serum has been confirmed through our study, and young serum-exo can be a valuable tool for treating HD.

\section{Abbreviations}

HD: Huntington's disease, mHtt: Mutant Huntingtin, NSC: Neural stem cell, SVZ: Subventricular zone

\section{Declarations}

\section{- Ethics approval}

All experimental animal procedures performed were approved by the Institutional Animal Care and Use Committee (IACUC, Approval number: 16-0043-C2A1) of Seoul National University Hospital, which was accredited by the Association for the Assessment and Accreditation of Laboratory Animal Care International.

\section{- Consent for publication}

Not applicable.

\section{- Availability of data and materials}

All data generated or analyzed during this study are included in this published article and its supplementary information files.

\section{- Competing interests}

The authors declare that they have no competing interests.

\section{- Funding}

This research was supported by the National Research Foundation of Korea (NRF) (2016M3C7A1914002, 2015R1D1A1A01060056)

\section{- Authors' contributions}


ML designed and conceptualized study, analyzed the data, and drafted the manuscript for intellectual content. WI and MK interpreted the data, and revised the manuscript for intellectual content. All authors read and approved the final manuscript.

\section{- Acknowledgements}

Not applicable.

\section{Reference}

1. Khakh BS, Sofroniew MV: Astrocytes and Huntington's disease. ACS Chem Neurosci 2014, 5:494-496.

2. Reddy PH: Increased mitochondrial fission and neuronal dysfunction in Huntington's disease: implications for molecular inhibitors of excessive mitochondrial fission. Drug Discov Today 2014, 19:951-955.

3. Bates GP, Dorsey R, Gusella JF, Hayden MR, Kay C, Leavitt BR, Nance M, Ross CA, Scahill RI, Wetzel R, et al: Huntington disease. Nat Rev Dis Primers 2015, 1:15005.

4. Smith MR, Syed A, Lukacsovich T, Purcell J, Barbaro BA, Worthge SA, Wei SR, Pollio G, Magnoni L, Scali $C$, et al: A potent and selective Sirtuin 1 inhibitor alleviates pathology in multiple animal and cell models of Huntington's disease. Hum Mol Genet 2014, 23:2995-3007.

5. Aronin N, DiFiglia M: Huntingtin-lowering strategies in Huntington's disease: antisense oligonucleotides, small RNAs, and gene editing. Mov Disord 2014, 29:1455-1461.

6. Ross CA, Aylward EH, Wild EJ, Langbehn DR, Long JD, Warner JH, Scahill RI, Leavitt BR, Stout JC, Paulsen JS, et al: Huntington disease: natural history, biomarkers and prospects for therapeutics. Nat Rev Neurol 2014, 10:204-216.

7. Hickey MA, Gallant K, Gross GG, Levine MS, Chesselet MF: Early behavioral deficits in R6/2 mice suitable for use in preclinical drug testing. Neurobiol Dis 2005, 20:1-11.

8. Kieburtz K, Reilmann R, Olanow CW: Huntington's disease: Current and future therapeutic prospects. Mov Disord 2018, 33:1033-1041.

9. Middeldorp J, Lehallier B, Villeda SA, Miedema SS, Evans E, Czirr E, Zhang H, Luo J, Stan T, Mosher $\mathrm{KI}$, et al: Preclinical Assessment of Young Blood Plasma for Alzheimer Disease. JAMA Neurol 2016, 73:1325-1333.

10. Menalled LB, Kudwa AE, Oakeshott S, Farrar A, Paterson N, Filippov I, Miller S, Kwan M, Olsen M, Beltran J, et al: Genetic deletion of transglutaminase 2 does not rescue the phenotypic deficits observed in R6/2 and zQ175 mouse models of Huntington's disease. PLoS One 2014, 9:e99520.

11. Li JY, Popovic N, Brundin P: The use of the R6 transgenic mouse models of Huntington's disease in attempts to develop novel therapeutic strategies. NeuroRx 2005, 2:447-464.

12. Katsimpardi L, Rubin LL: Young systemic factors as a medicine for age-related neurodegenerative diseases. Neurogenesis (Austin) 2015, 2:e1004971. 
13. Villeda SA, Plambeck KE, Middeldorp J, Castellano JM, Mosher KI, Luo J, Smith LK, Bieri G, Lin K, Berdnik D, et al: Young blood reverses age-related impairments in cognitive function and synaptic plasticity in mice. Nat Med 2014, 20:659-663.

14. Wyss-Coray T: Ageing, neurodegeneration and brain rejuvenation. Nature 2016, 539:180-186.

15. Allison LI, Suzie C: Exosomes: The Messengers of Health and Disease. Current Neuropharmacology 2017, 15:157-165.

16. Kalani A, Tyagi A, Tyagi N: Exosomes: mediators of neurodegeneration, neuroprotection and therapeutics. Mol Neurobiol 2014, 49:590-600.

17. S ELA, Mager I, Breakefield XO, Wood MJ: Extracellular vesicles: biology and emerging therapeutic opportunities. Nat Rev Drug Discov 2013, 12:347-357.

18. Cho YE, Mezey E, Hardwick JP, Salem N, Jr., Clemens DL, Song BJ: Increased ethanol-inducible cytochrome P450-2E1 and cytochrome P450 isoforms in exosomes of alcohol-exposed rodents and patients with alcoholism through oxidative and endoplasmic reticulum stress. Hepatol Commun 2017, 1:675-690.

19. Boukouris S, Mathivanan S: Exosomes in bodily fluids are a highly stable resource of disease biomarkers. Proteomics Clin App/ 2015, 9:358-367.

20. Keller S, Sanderson MP, Stoeck A, Altevogt P: Exosomes: from biogenesis and secretion to biological function. Immunol Lett 2006, 107:102-108.

21. Ludwig AK, Giebel B: Exosomes: small vesicles participating in intercellular communication. Int $J$ Biochem Cell Biol 2012, 44:11-15.

22. Lee M, Liu T, Im W, Kim M: Exosomes from adipose-derived stem cells ameliorate phenotype of Huntington's disease in vitro model. European Journal of Neuroscience 2016, 44:2114-2119.

23. Im W, Ban J, Lim J, Lee M, Lee S-T, Chu K, Kim M: Extracts of adipose derived stem cells slows progression in the R6/2 model of Huntington's disease. PloS one 2013, 8:e59438-e59438.

24. Hoffman E, Pickavance L, Thippeswamy T, Beynon RJ, Hurst JL: The male sex pheromone darcin stimulates hippocampal neurogenesis and cell proliferation in the subventricular zone in female mice. Frontiers in Behavioral Neuroscience 2015, 9.

25. Rimer JM, Lee J, Holley CL, Crowder RJ, Chen DL, Hanson PI, Ory DS, Schaffer JE: Long-range function of secreted small nucleolar RNAs that direct 2'-0-methylation. Journal of Biological Chemistry 2018, 293:13284-13296.

26. Jiang Q, Oldenburg R, Otsuru S, Grand-Pierre AE, Horwitz EM, Uitto J: Parabiotic Heterogenetic Pairing of Abcc6-/-/Rag1-/- Mice and Their Wild-Type Counterparts Halts Ectopic Mineralization in a Murine Model of Pseudoxanthoma Elasticum. The American Journal of Pathology 2010, 176:1855-1862.

27. Bu XL, Xiang Y, Jin WS, Wang J, Shen LL, Huang ZL, Zhang K, Liu YH, Zeng F, Liu JH, et al: Bloodderived amyloid- $\beta$ protein induces Alzheimer's disease pathologies. Molecular Psychiatry 2018, 23:1948-1956. 
28. Horowitz A, Villeda S: Therapeutic potential of systemic brain rejuvenation strategies for neurodegenerative disease [version 1; peer review: 3 approved]. F1000Research 2017, 6.

29. Janas AM, Sapoń K, Janas T, Stowell MHB, Janas T: Exosomes and other extracellular vesicles in neural cells and neurodegenerative diseases. Biochimica et Biophysica Acta (BBA) - Biomembranes 2016, 1858:1139-1151.

30. Kamerkar S, LeBleu VS, Sugimoto H, Yang S, Ruivo CF, Melo SA, Lee JJ, Kalluri R: Exosomes facilitate therapeutic targeting of oncogenic KRAS in pancreatic cancer. Nature 2017, 546:498-503.

31. Colao IL, Corteling R, Bracewell D, Wall I: Manufacturing Exosomes: A Promising Therapeutic Platform. Trends in Molecular Medicine 2018, 24:242-256.

\section{Figures}


a Schematics of parabiotic paring
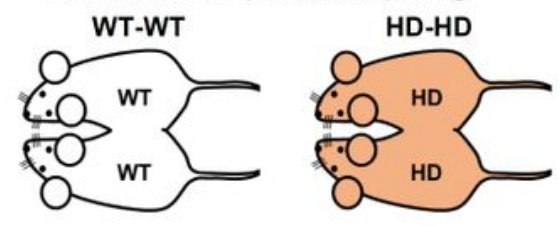

b Injection of BrdU to parabionts
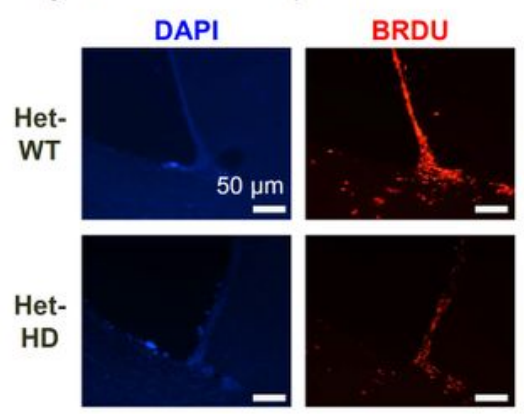

d Survival of parabiotic pairs

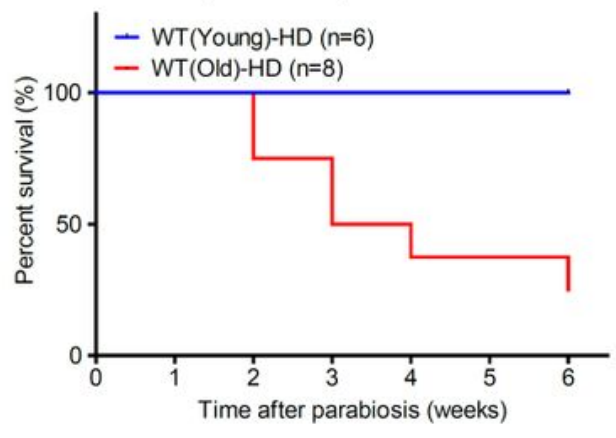

e Body weight of parabiotic pairs

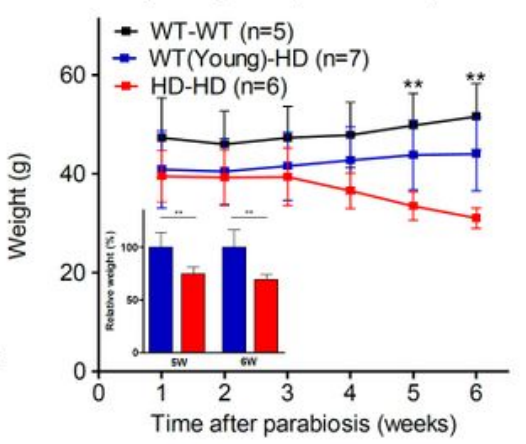

f $\mathrm{mHtt}$ aggregation of parabionts in striatum and cortex
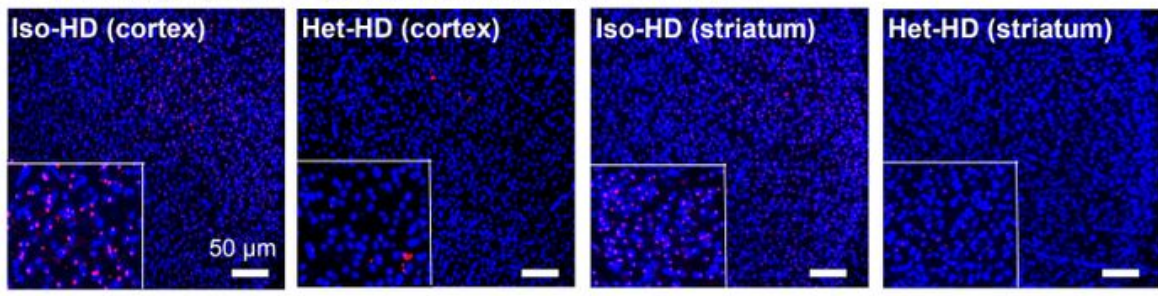

g $\mathrm{mHtt}$ aggregation in proteins extracted from the brain
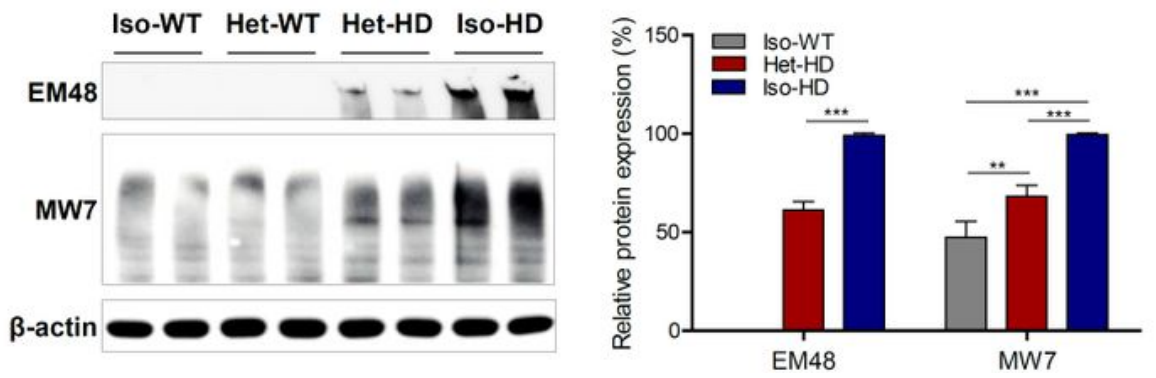

\section{Figure 1}

Construction of animal model of heterochronic parabiosis for HD and modulation of HD pathology.a Schematics of parabiotic paring. WT-WT indicates the isochronic paring of wild-types, HD-HD indicates the isochronic paring of HD mouse, and each parabionts are indicated as Iso-WT and Iso-HD, respectively. WT(Young)-HD indicates the heterochronic paring of young wild-type and HD mouse, which are indicated as Het WT and Het HD, respectively, and WT(Old)-HD indicates the heterochronic paring of old wild-type 
and HD mouse. b Analysis of BrdU signal for WT(Young)-HD with the injection of BrdU to the abdomen of Het-WT. c Comparison of the genotype of DNA from the Het-WT with that of the WT and the HD mice. $d$ Effect of the age of wild-type on survival of HD mice paired with the wild-type. e Analysis of body weight of parabionts. Inset is relative comparison of the body weight of WT(Young)-HD and HD-HD. $\mathrm{f} \mathrm{mHtt}$ aggregation in striatum and cortex for Iso-HD and Het-HD. g Evaluation of mHtt aggregation from the proteins in the brain of parabionts by western blot.

a Improvement of mitochondrial dysfunction
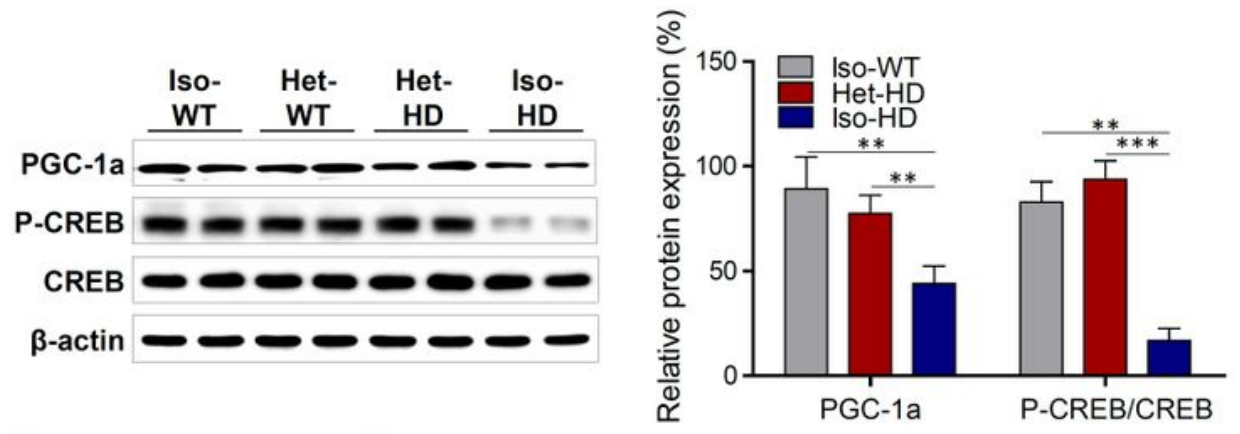

b Modulations of cell death
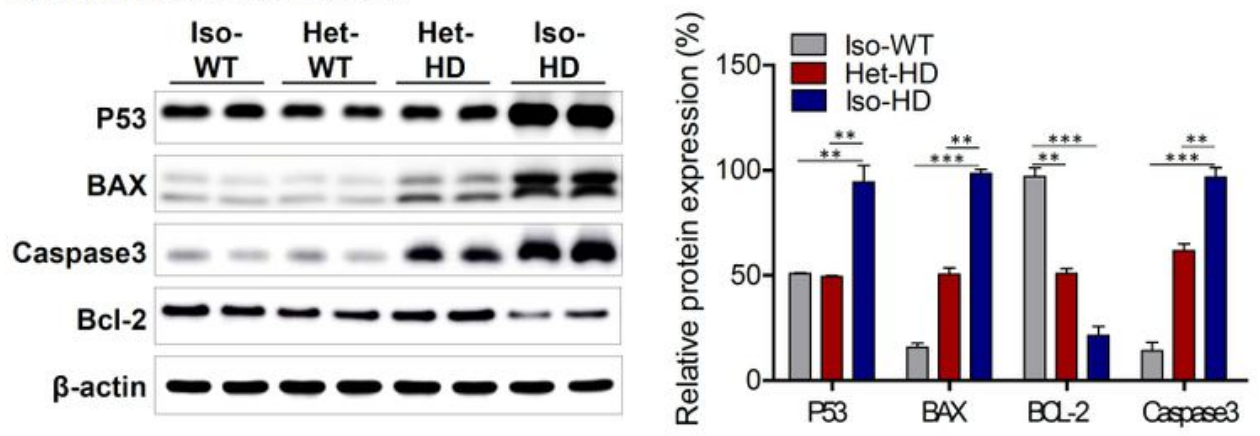

C Immunohistochemistry of parabiotic individuals

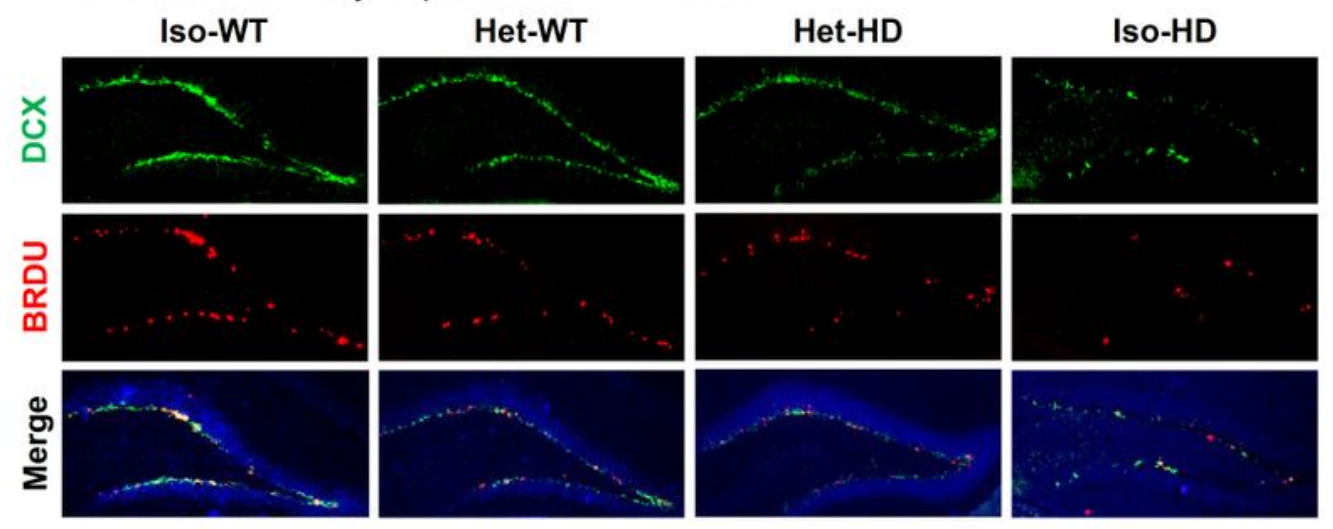

d Western blot of parabiotic individuals
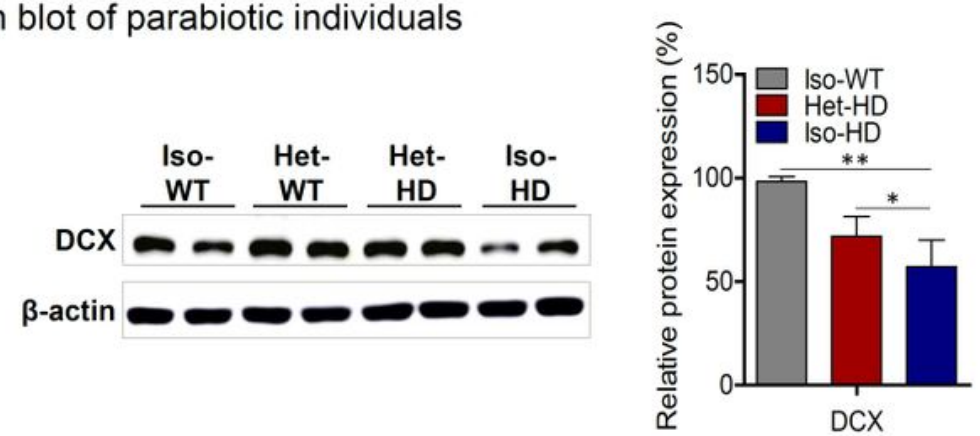

Figure 2 
Modulation of pathological phenotypes of HD by parabiosis. a Improvement of mitochondrial dysfunction.b Modulation of cell death. c, d Immunohistochemistry and western blot of parabionts to demonstrate cognitive function of parabionts, respectively.

a Exosomes derived from young and old serum
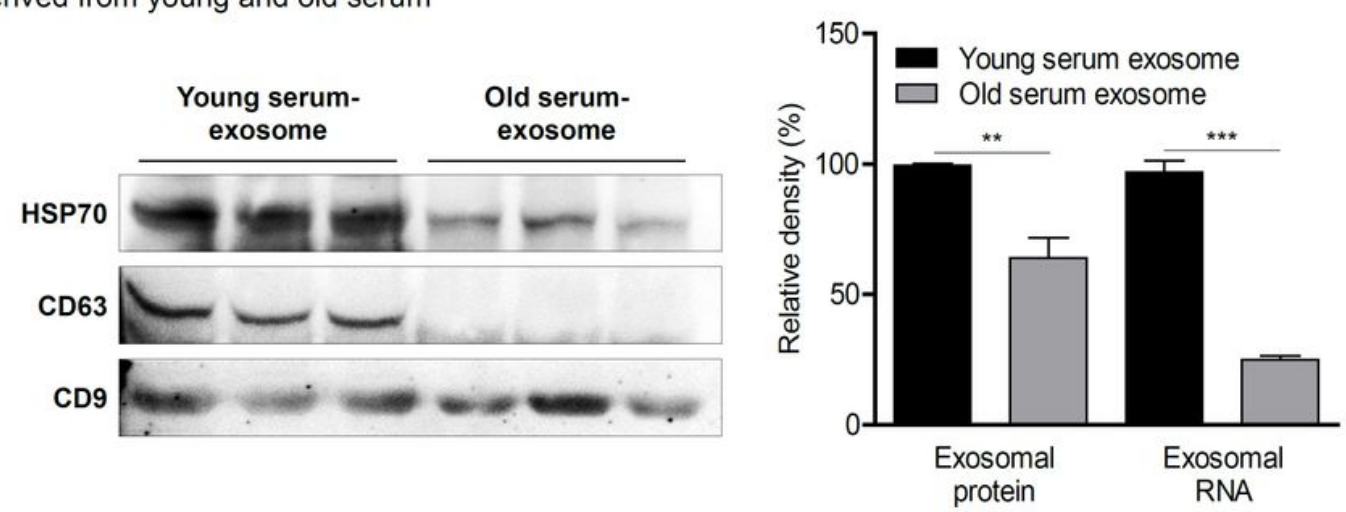

b Amelioration of $\mathrm{mHtt}$ aggregation by exosome treatment

CTL

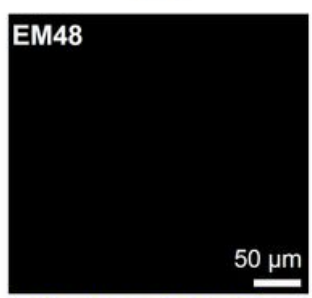

Merge
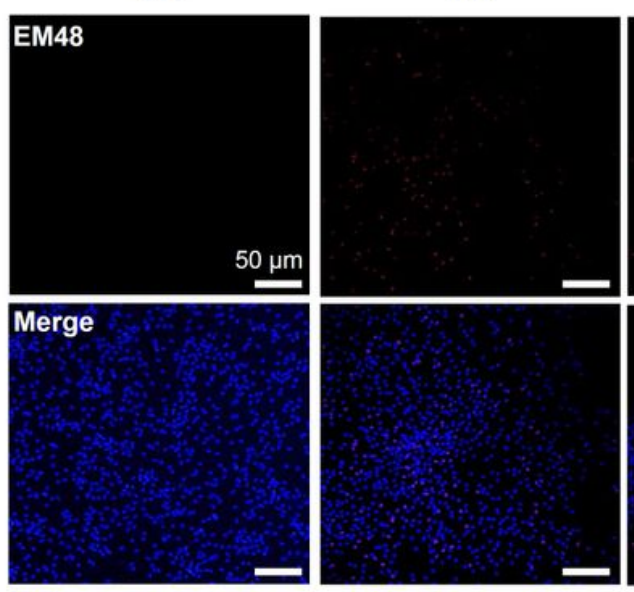

HD + Young
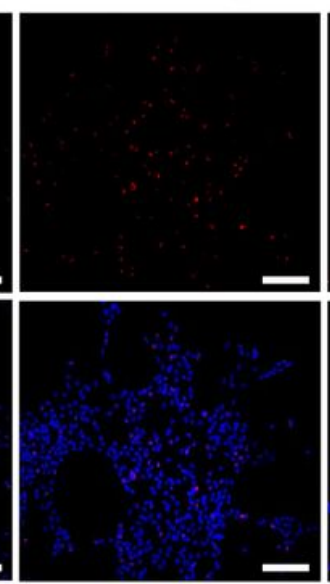

HD + Young-exo
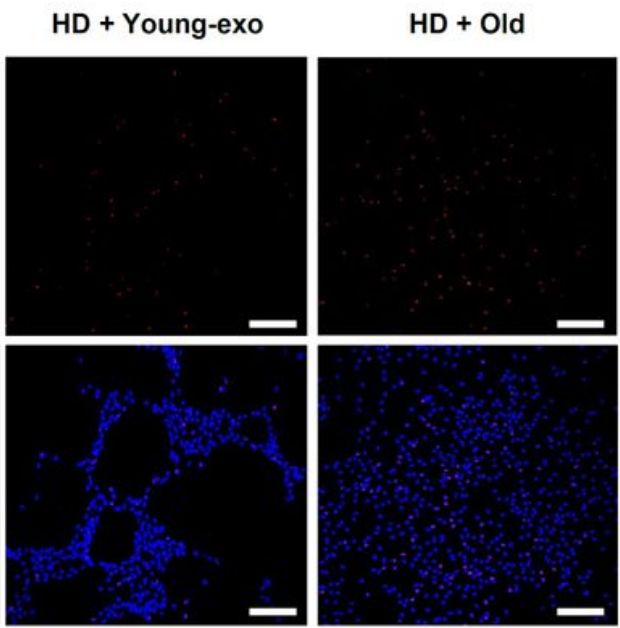

C Western blot analysis for exosome treatment
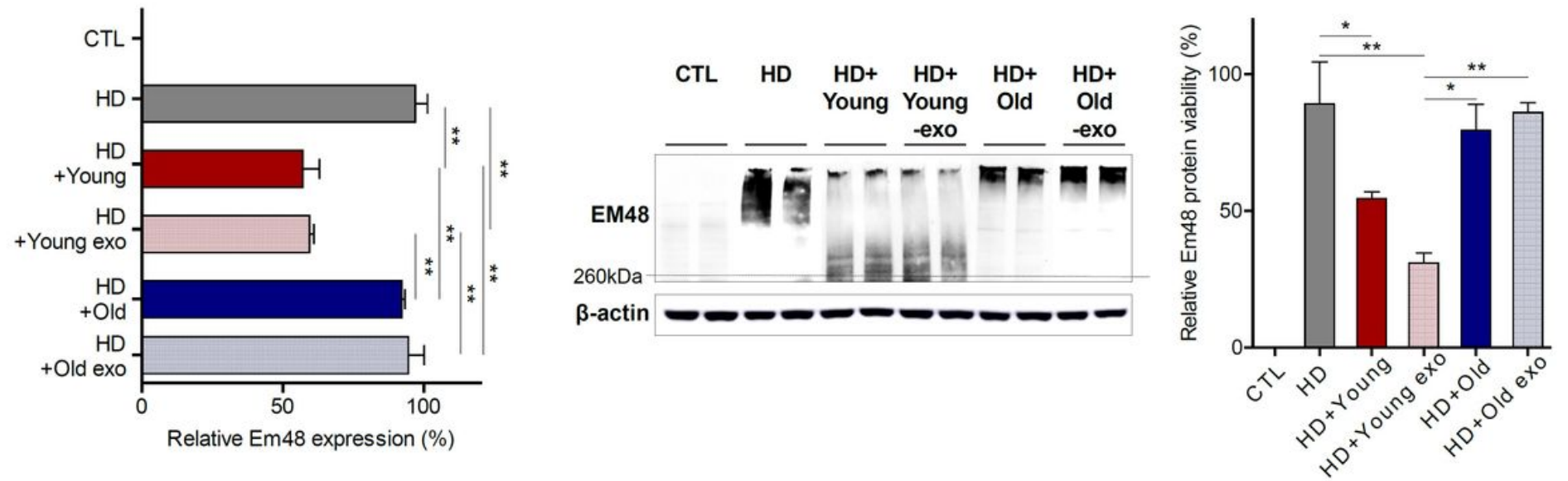

\section{Figure 3}

Amelioration of $\mathrm{mHtt}$ aggregation by exosome treatment.a Comparison of the amount of exosomes, exosomal proteins, and RNA levels of blood serum from young and old wild-type. Young serum-exosome and Old serum-exosome indicates the exosomes derived from the blood serum from the young mice and old mice, respectively. b, clmmunohistochemistry and western blot of cells from demonstratemHtt 
aggregates in cells. CTL indicates the cells from the wild-type mice, and HD indicates the cells from the $\mathrm{HD}$ mice. $\mathrm{HD}+$ Young and HD+Young-exo indicates the HD cells treated by blood serum and exosomes derived by blood serum from the young wild-type mice, respectively. $\mathrm{HD}+\mathrm{Old}$ and $\mathrm{HD}+\mathrm{Old}$-exo indicates the HD cells treated by blood serum and exosomes derived by blood serum from the old wild-type mice, respectively.

a Improvement of mitochondrial dysfunction

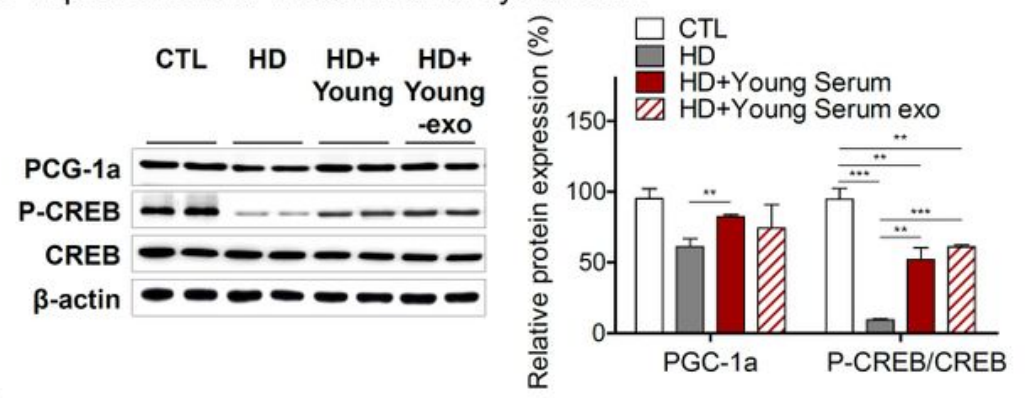

b Modulations of cell death

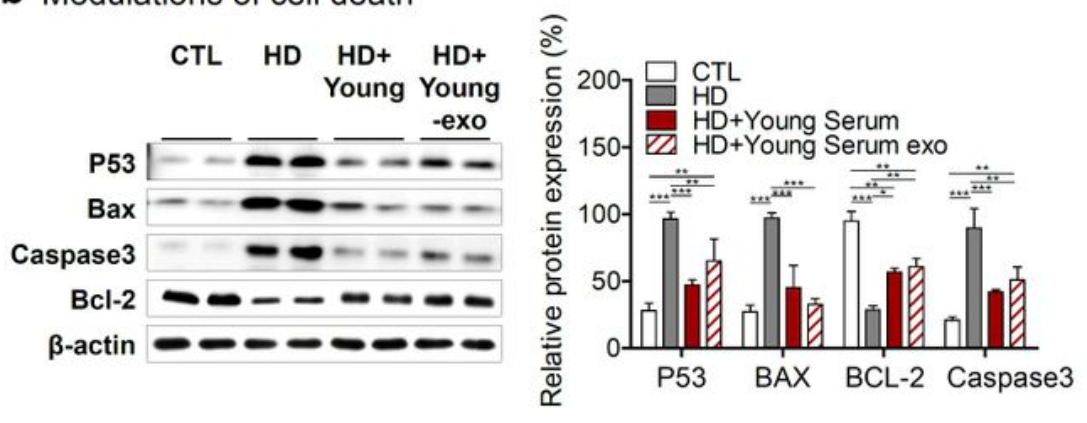

C Analysis for cell death and cell proliferation

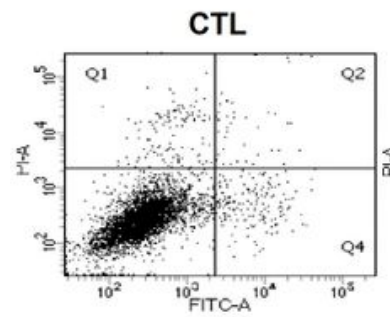

$\mathrm{HD}+$ Young
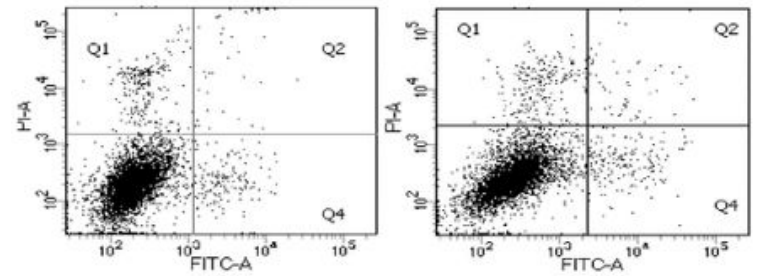

d Evaluation of cell viability with exsome treatment

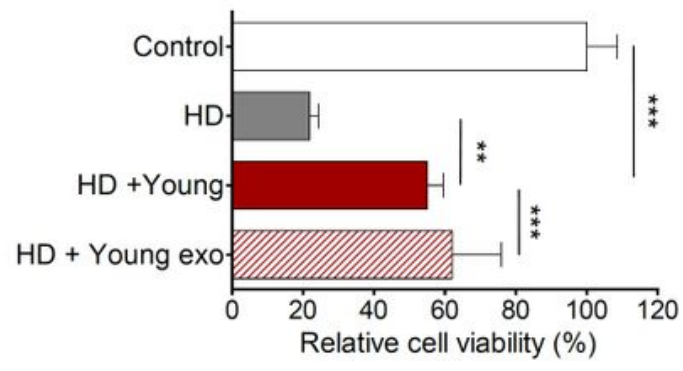

Figure 4

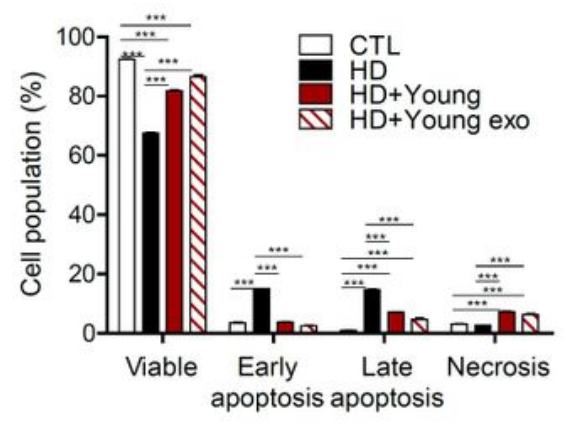


Modulation of molecular pathology of HD by exosome treatment.a, b Improvement of mitochondrial dysfunction and modulation cell death, respectively. c Analysis for cell death. $d$ Evaluation of cell viability with exosome treatment.

\section{Supplementary Files}

This is a list of supplementary files associated with this preprint. Click to download.

- FigureS8.docx

- Figures7.docx

- FigureS6.docx

- FigureS5.docx

- FigureS4.docx

- Figures3.docx

- Video11.mp4

- Video10.mp4

- Video9.mp4

- Video8.mp4

- Video7.mp4

- Video6.mp4

- Video5.mp4

- Video4.mp4

- Video3.mp4

- Video2.mp4

- Video1.mp4

- Figures2.docx

- FigureS1.docx 